\title{
The structure of paranoia in the general population
}

Paul E. Bebbington, Orla McBride, Craig Steel, Elizabeth Kuipers, Mirjana Radovanovič, Traolach Brugha, Rachel Jenkins, Howard I. Meltzer and Daniel Freeman

\section{Background}

Psychotic phenomena appear to form a continuum with normal experience and beliefs, and may build on common emotional interpersonal concerns.

\begin{abstract}
Aims
We tested predictions that paranoid ideation is exponentially distributed and hierarchically arranged in the general population, and that persecutory ideas build on more common cognitions of mistrust, interpersonal sensitivity and ideas of reference.

\section{Method}

Items were chosen from the Structured Clinical Interview for DSM-IV Axis II Disorders (SCID-II) questionnaire and the Psychosis Screening Questionnaire in the second British National Survey of Psychiatric Morbidity $(n=8580)$, to test a putative hierarchy of paranoid development using confirmatory factor analysis, latent class analysis and factor mixture modelling analysis.
\end{abstract}

\section{Results}

Different types of paranoid ideation ranged in frequency from less than $2 \%$ to nearly $30 \%$. Total scores on these items followed an almost perfect exponential distribution $(r=0.99)$. Our four a priori first-order factors were corroborated (interpersonal sensitivity; mistrust; ideas of reference; ideas of persecution). These mapped onto four classes of individual respondents: a rare, severe, persecutory class with high endorsement of all item factors, including persecutory ideation; a quasi-normal class with infrequent endorsement of interpersonal sensitivity, mistrust and ideas of reference, and no ideas of persecution; and two intermediate classes, characterised respectively by relatively high endorsement of items relating to mistrust and to ideas of reference.

\section{Conclusions}

The paranoia continuum has implications for the aetiology, mechanisms and treatment of psychotic disorders, while confirming the lack of a clear distinction from normal experiences and processes.

\section{Declaration of interest}

None.
Paranoid ideation is characteristic of psychotic illness, but, like other psychotic phenomena, may be widespread in non-clinical populations. $^{1-5}$ Wariness of the intentions of others may be adaptive in some situations, and becomes a clinical problem only when it is excessive, exaggerated or distressing, or interferes with functioning. Given that such ideation may precede delusion formation, ${ }^{6,7}$ our understanding of delusions should be enhanced by studying paranoid thinking in non-clinical populations. In our cognitive model of persecutory delusions, ${ }^{8}$ we hypothesised that even severe paranoia builds on common emotional concerns, particularly themes of interpersonal worry or social anxiety. The interpersonal sensitivities often seen in emotional disorders (e.g. concerns about rejection or about being vulnerable) inform worries about future threat and the intention of others. In some people, these fears lead to ideas that others are watching or talking about them. Ideas of persecution are hypothesised to emerge from these ideas of reference. This process implies a close structured relationship between worry, anxiety and paranoia. It has credence, as all concern the theme of the anticipation of threat, ${ }^{9}$ and there is increasing empirical evidence for links between affect and paranoia. ${ }^{10-15}$

The current investigation employs data from a general population survey to examine the distribution and underlying structure of components of paranoid ideation. Our analyses were driven by the hypothesis that the overall distribution of such ideation should be similar in form to that of affective symptoms, with many people having few such thoughts and a few people having many. 3,16,17 Moreover, as with affective symptoms, increasing symptom counts should be characterised by the recruitment of rarer and odder ideas: ${ }^{18}$ in other words, a hierarchy of paranoid thoughts underpins an inherent structure within the continuum. In our cognitive model of paranoia, ${ }^{8}$ we postulated four subcategories of paranoid experience: interpersonal sensitivities; mistrust; ideas of reference; and ideas of persecution. Moreover, we postulate that this structure arises because the subcategories are linked as part of a hierarchical process. Members of the general population would be classifiable in terms of these factors, and the resulting classification would correspondingly reflect hierarchical relationships between the factors.

These ideas can be formally tested in a number of ways. Given our specific hypotheses, three were appropriate: (1) confirmatory factor analysis (CFA), which establishes the structure of linear relationships between items; (2) latent class analysis (LCA), which attributes individuals to a number of separate classes; and (3) the hybrid procedure, factor mixture modelling analysis (FMMA), which allows the factor structure to be related to the class structure.

\section{Method}

The second British National Psychiatric Morbidity Survey was carried out on a representative sample of the British population in $2000 .^{19,20}$

\section{Sample}

Because of its good coverage, the British small users postcode address file was used to generate a list of private households. ${ }^{19}$ Full details of sampling are provided elsewhere. ${ }^{20}$ A total of 15804 addresses were obtained. Interviewers visited these to identify 
private households with at least one person aged 16-74 years. One person was selected from each qualifying household using the Kish grid method. ${ }^{21}$ Just under $70 \%$ of those approached agreed to a first phase interview, which the vast majority completed in full, providing 8576 individuals for the current analysis.

\section{Design}

The survey comprised two phases. ${ }^{19,20}$ The first was carried out by well-trained non-clinical interviewers employed by the Office for National Statistics. It included the use of screening instruments to determine participants for a further interview by trained clinicians to establish diagnoses of psychosis and personality disorder. To test our hypotheses, we abstracted items from two screening instruments: the Psychosis Screening Questionnaire $(\mathrm{PSQ})^{22}$ and the questionnaire version of the Structured Clinical Interview for DSM-IV Axis II Disorders (SCID-II). ${ }^{23}$ We used data from the 2000 British National Psychiatric Morbidity Survey as it is the only one in the British national survey programme to include both these measures. ${ }^{24}$

\section{Measuring paranoia}

For current purposes, we used items 2, 3, 3a and 3b from the PSQ, relating to ideas of persecution, conspiracy and interference. From the SCID-II, we used items 2, 3, 4, 6, 10, 25, 26, 27, 28, 33 and 35. These relate to different personality disorders (avoidant, dependent, paranoid and schizotypal), but were chosen a priori in line with our specific interest in cognitive content (progressing from mistrust through reference to persecution). This enabled us to test our ideas of how paranoia builds on certain common worries. Our 15 selected items approximate to those used by Freeman et al. ${ }^{3}$ On theoretical grounds, they were separated into four groups: mistrust, interpersonal sensitivities, ideas of reference, and ideas of persecution (see Table 1). We also summed the item endorsements to produce an overall paranoia score for each individual. Finally, for each item, we calculated the mean difference for the total item count between those with and those without the given item (correcting for the contribution due to that item). ${ }^{18}$ We term this the excess item score.

\section{Strategy of analysis}

We first analysed the frequency distribution of individual paranoia scores in our total sample. We hypothesised that, as with affective symptoms,${ }^{16}$ this distribution would be exponential.

We used Mplus version $6^{25}$ for Windows to conduct the latent variable modelling. Confirmatory factor analysis is used to test specific hypotheses about the content and the number of dimensions (factors) that underlie a set of variables purporting to measure a given phenomenon (here paranoid ideation). The resulting factors help to explain the nature of the interrelationships between the observed variables (the individual paranoia items). Latent class analysis, in contrast, explores whether individuals can be classified into groups (classes) based on their particular endorsement of the paranoia items. The basic difference between the two procedures lies in the underlying latent variable - in CFA, paranoia is viewed as a continuous latent variable, whereas in LCA, it is categorical. Hybrid models, as in FMMA, incorporate both continuous and categorical latent variables - these models propose that there may be one (or more) dimension(s) of paranoia, and at different points along the dimension(s), there are groups of people (classes) who are homogeneous within class, and heterogeneous across classes, in relation to their paranoia symptoms. Hybrid models can be estimated in a variety of different ways, and the exact make-up of each model should be based on pre-existing theory, of the type we test here.

Three CFA models were tested: (1) a one-factor model representing 'severity of paranoid ideation'; (2) a four-factor model representing our a priori factors 'interpersonal sensitivities', 'mistrust', 'ideas of reference' and 'ideas of persecution'; and (3) a higher-order factor model, representing a second-order factor of 'severity of paranoid ideation' underpinned by the four first-order factors outlined in the previous model. Figure 1 illustrates these models. The ovals represent the latent variables or factors and

\begin{tabular}{|c|c|c|c|}
\hline Item & Question & $\begin{array}{l}\text { Frequency } \\
\%\end{array}$ & $\begin{array}{l}\text { Excess } \\
\text { item score }\end{array}$ \\
\hline \multicolumn{4}{|c|}{ Interpersonal sensitivities } \\
\hline PD2 & Do you avoid getting involved with people unless you are certain they will like you? & 12.6 & 3.56 \\
\hline PD4 & Do you often worry about being criticised or rejected in social situations? & 27.7 & 3.07 \\
\hline PD6 & Do you believe that you're not as good, as smart, or as attractive as most other people? & 25.4 & 2.49 \\
\hline PD10 & Do you find it hard to disagree with people even when you think they are wrong? & 20.3 & 1.97 \\
\hline \multicolumn{4}{|l|}{ Mistrust } \\
\hline PD3 & Do you find it hard to be 'open' even with people you are close to? & 20.8 & 2.44 \\
\hline PD26 & Do you spend a lot of time wondering if you can trust your friends or the people you work with? & 15.5 & 3.96 \\
\hline PD25 & Do you often have to keep an eye out to stop people from using you or hurting you? & 28.1 & 2.70 \\
\hline PD27 & $\begin{array}{l}\text { Do you find that it is best not to let other people know much about you because they will use } \\
\text { it against you? }\end{array}$ & 21.3 & 3.13 \\
\hline \multicolumn{4}{|c|}{ Ideas of reference } \\
\hline PD28 & Do you often detect hidden threats or insults in things people say or do? & 19.0 & 3.57 \\
\hline PD33 & When you are out in public and see people talking, do you often feel that they are talking about you? & 6.5 & 5.07 \\
\hline PD35 & When you are around people, do you often get the feeling that you are being watched or stared at? & 9.7 & 4.69 \\
\hline \multicolumn{4}{|c|}{ Ideas of persecution } \\
\hline PSQ3 & Over the past year, have there been times when you felt that people were against you? & 20.9 & 3.00 \\
\hline PSQ2 & $\begin{array}{l}\text { Have you ever felt that your thoughts were directly interfered with or controlled by some outside } \\
\text { force or person? }\end{array}$ & 9.0 & 2.03 \\
\hline PSQ3a & $\begin{array}{l}\text { Have there been times when you felt that people were deliberately acting to harm you or your } \\
\text { interests? }\end{array}$ & 9.0 & 3.98 \\
\hline PSQ3b & $\begin{array}{l}\text { Have there been times you felt that a group of people was plotting to cause you serious harm or } \\
\text { injury? }\end{array}$ & 1.5 & 5.52 \\
\hline
\end{tabular}



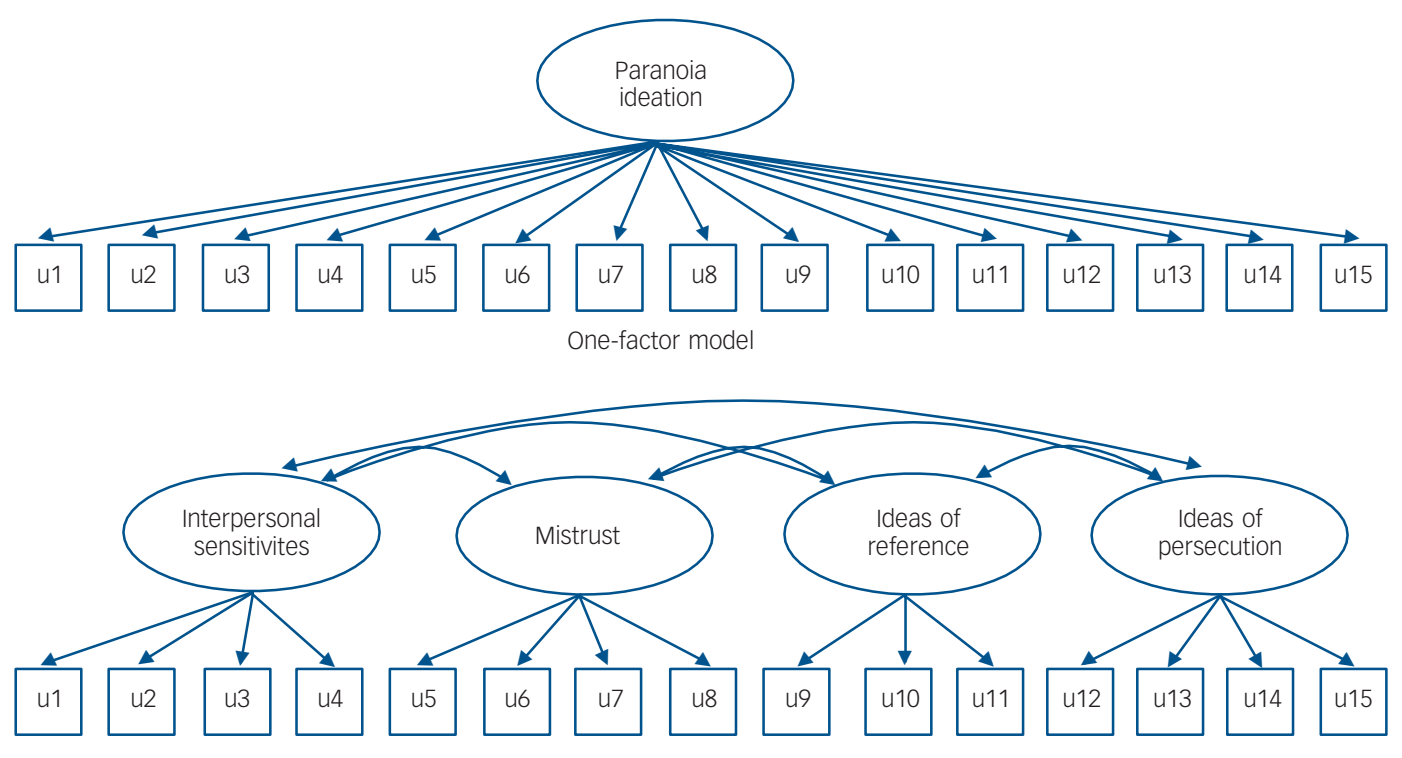

Four-factor first-order model

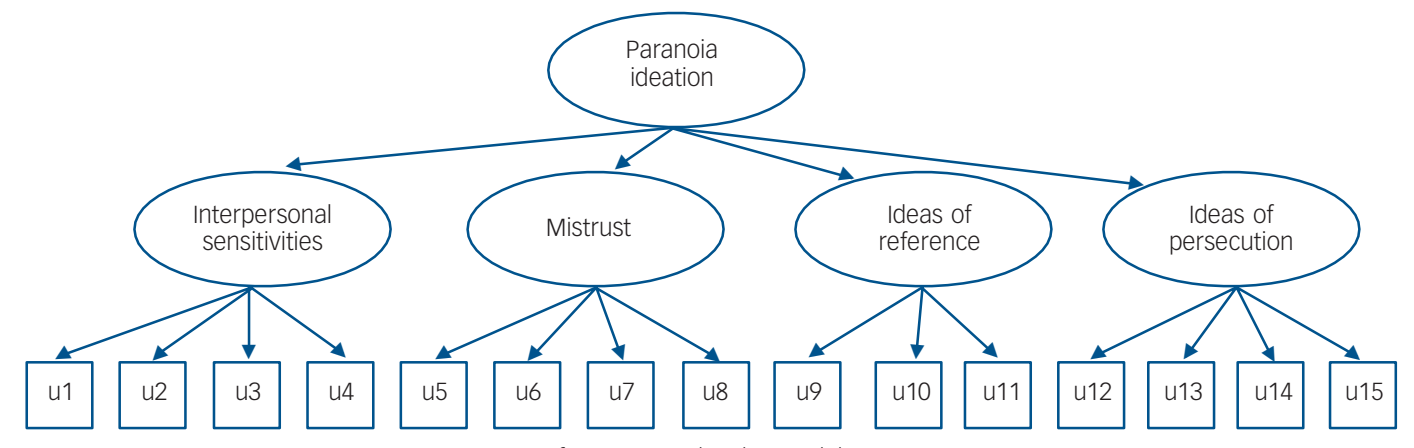

Four-factor second-order model

Fig. 1 Alternative factor models conceptualising the dimensionality of paranoia items.

the square boxes represent observed binary variables. The arrows connecting the factors to the categorical indicators ( $\mathrm{u} 1-\mathrm{u} 15)$ represent factor loadings. The curved connections between the factors represent correlations. The default estimator for this analysis was a robust maximum likelihood (WLSMV) estimator. All factor loadings were estimated, with factor variances fixed at one.

Next, a series of successive latent class models, varying the number of classes from 1 to 8 , were estimated. Latent class analysis evaluates whether a group of associated observed variables can be related to an underlying categorical variable, comprising two or more classes (levels). ${ }^{26}$ Latent class analysis, as used here, allowed us to consider not only the number of items endorsed, but also their overall pattern of distribution. ${ }^{27}$ Decisions regarding the most appropriate model should be guided both by statistical fit indices and by conceptual considerations, that is, the meaningfulness and distinctiveness of the latent class profiles. The default estimator for this analysis was a robust maximum likelihood (MLR) estimator. Further details outlining how these models were estimated and evaluated are provided in the online supplement.

One limitation of LCA is that it fails to account for individual difference within classes. Factor mixture modelling analysis ${ }^{28}$ combines the latent class model and the common factor model, and has a single categorical, and one or more continuous, latent variables. In FMMA, continuous latent variables are used to explain the variation and covariation in a set of observed items, while the categorical latent variable represents heterogeneity at the factor level. ${ }^{27}$ Thus, the superiority of factor mixture models over other conventional models is that they permit simultaneous classification of people into diagnostic groups, while also modelling the severity of disorder. ${ }^{29}$ Use of FMMA models is increasing in psychiatry. However, it is still relatively uncommon, and procedural conventions are not yet fully established. In the current study we chose to estimate five different model types, varying in terms of restrictiveness, as outlined by Clark et $a l^{29}$ (Table 2).

Sampling weights and other variables that account for the complex survey design of National Psychiatric Morbidity Survey 2000 were used in all analyses to enhance the reliability and validity of the parameter estimates, standard error and model fit calculations. The default estimator for the FMMA was an MLR estimator.

\section{Results}

\section{The prevalence of individual paranoia items}

The endorsement of individual paranoia items was considerable, ranging from $1.5 \%$ to $28 \%$ (Table 1 ).

The total number of paranoia items could range from 0 to 15, although in the event no one scored 15 (weighted mean 2.3, s.d. =2.6). The distribution of total scores is displayed in Fig. 2. The data could be fitted by a single continuous distribution model (16), following an exponential curve with a correlation between observed and model estimated scores of 0.99 . 


\begin{tabular}{|c|c|c|c|c|c|c|}
\hline Restrictiveness & Model & Factor variance & Factor covariance & Factor mean & Factor loadings & Item thresholds (latent classes) \\
\hline \multirow[t]{4}{*}{ Most } & FMM-1 & Fixed at zero & Fixed at zero & $\begin{array}{l}\text { Varies across } \\
\text { classes }\end{array}$ & Equal across classes & Equal across classes \\
\hline & FMM-2 & Freely estimated & Freely estimated & Set to zero & Equal across classes & Equal across classes \\
\hline & FMM-3 & $\begin{array}{l}\text { Freely estimated, but } \\
\text { equal across classes }\end{array}$ & $\begin{array}{l}\text { Freely estimated, but } \\
\text { equal across classes }\end{array}$ & Set to zero & Equal across classes & Allowed to vary across classes \\
\hline & FMM-4 & $\begin{array}{l}\text { Allowed to change } \\
\text { across classes }\end{array}$ & $\begin{array}{l}\text { Allowed to change } \\
\text { across classes }\end{array}$ & Set to zero & Equal across classes & Allowed to vary across classes \\
\hline Least & FMM-5 & $\begin{array}{l}\text { Allowed to change } \\
\text { across classes }\end{array}$ & $\begin{array}{l}\text { Allowed to change } \\
\text { across classes }\end{array}$ & Set to zero & $\begin{array}{l}\text { Allowed to vary across } \\
\text { classes }\end{array}$ & Allowed to vary across classes \\
\hline
\end{tabular}

\section{Non-reflexive relationships between items}

As predicted, positive excess item scores were associated with each item, confirming that the relationship between items was not random (Table 1). The mean excess score was 3.5 (s.d.=1.0). However, the excess score varied between the items, ranging from 2.0 to 5.5. If the relationship between items is non-reflexive (i.e. their endorsement follows a hierarchical arrangement), the rarer items should be associated with a greater excess score than the more frequent ones. This is what we found. Thus, the excess symptom score associated with each item was significantly and strongly associated with the frequency of endorsement of that item $(r=-0.79, P<0.001)$. For example, worry about people using or hurting the participant (frequency 28\%) was associated with an excess symptom score of 2.7 , whereas a belief about plots designed to cause serious harm (frequency $1.5 \%$ ) was associated with an excess symptom score of 5.5.

\section{Confirmatory factor analysis}

Table 3 outlines the standardised factor loadings, factor correlations and goodness-of-fit indices for the competing CFA models. Despite moderate to strong factor loadings, the one-factor model did not generally fit the data well. Both the first- and second-order four-factor models provided a good fit to the data, with strong factor loadings (first-order ranging 0.514-0.978; second-order ranging 0.629-0.953). A chi-squared difference test for nested models (see online supplement) revealed that the firstorder four-factor model was a superior fit to the one-factor model $\left(\chi^{2}\right.$ diff $=1245.355$, d.f. diff $\left.=6, P<0.001\right)$ and the second-order four-factor model $\left(\chi^{2}\right.$ diff $=24.656$, d.f. diff $\left.=2, \quad P<0.001\right)$.

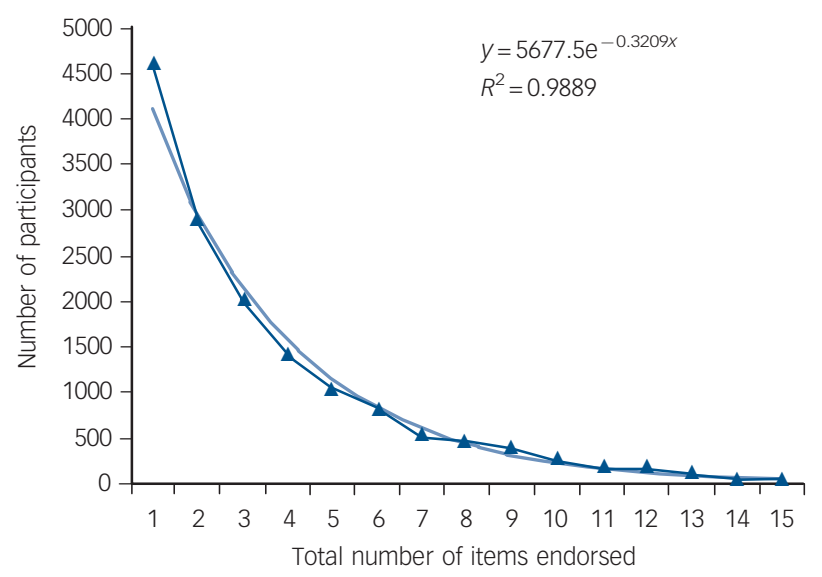

Fig. 2 The distribution of total paranoia scores in the general population.
Collectively, these model results provided strong confirmation of our a priori grouping of items.

\section{Latent class analysis}

The results for the competing latent class models are presented in Table 4 . The fit indices did not identify clearly which model provided the best explanation of the data (the log-likelihood value, Akaike and Bayesian information criteria and sample size adjusted Bayesian information criterion continued to decrease as the number of classes in the models increased). This was not unexpected, given that we had hypothesised a priori that underlying the categorisation of classes is a dimension of severity. This is not captured by LCA, but can be modelled using FMMA.

\section{Factor mixture modelling analysis}

This is often useful in reducing the number of classes into more meaningful subgroups, especially if the classes are modelling differences in severity. The FMMA results presented in Table 5 should be interpreted in the light of our theoretical model of paranoia, specifically that the items are related non-reflexively, with the more extreme paranoia items being associated with a greater overall severity, as indicated by the item count. Based on the goodness-of-fit indices, two models stand out (shown in bold in Table 5): both were one-factor models with four latent classes. Following the notation of Clark et al, ${ }^{29}$ the best-fitting model in terms of the Bayesian information criterion was the four-class variant of FMM-3 (FMM-3, 4C). This model proposes that: (1) people in the survey can be categorised into four groups (or classes): the people in each class experience a similar type of paranoid ideation, distinct from that experienced by people in the other classes; and (2) underlying each class, there is a single dimension of 'paranoia', which is conceptualised identically in each class (as indicated by the invariant factor loadings; range of standardised loadings $0.394-0.850$ ). In other words, the level of paranoia ('severity') is the same in each class (as indicated by the invariant factor variance). On both theoretical and empirical grounds, this assumption is, however, implausible - people in the community with different types of paranoid experiences will vary in terms of the severity of those experiences.

We therefore considered model $4 \mathrm{C}$ of the FMM-4 type to be the best conceptual model overall. This provided a good explanation of the data, very similar to its FMM-3 equivalent, and was less restrictive, in that the factor variances were allowed to vary across classes. This implies differences in terms of the severity of paranoid ideation, both between classes and within each class, i.e. between the class members (see Muthén \& Asparouhove ${ }^{30}$ ).

The estimated probabilities for the paranoia items derived from model FMM-4 are illustrated in Fig. 3. The items are 
Table 3 Standardised factor loadings, factor correlations, and goodness-of-fit statistics for three competing confirmatory factor analytic models of 15 paranoia items from 2000 British National Psychiatric Morbidity Survey $(n=8576)$

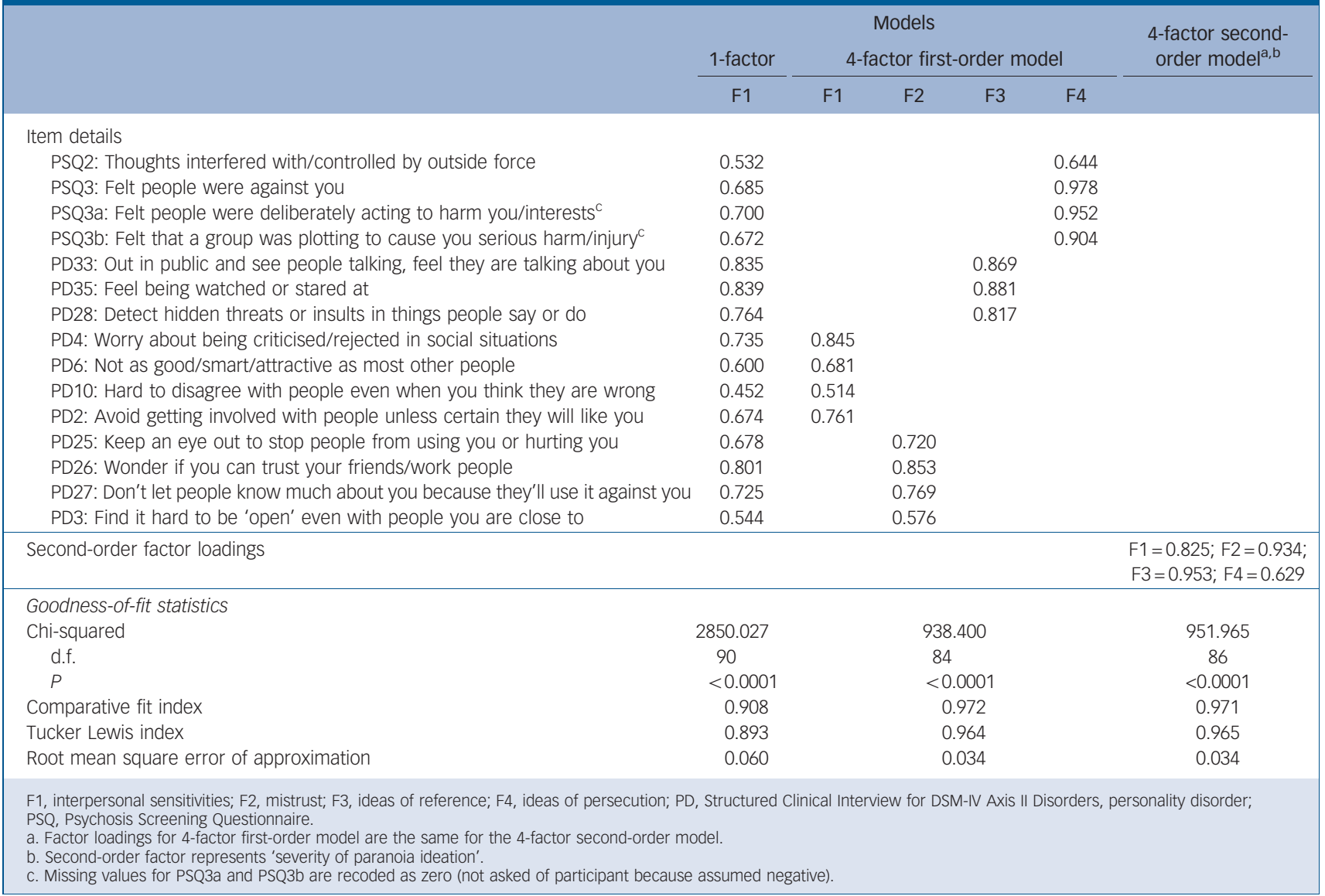

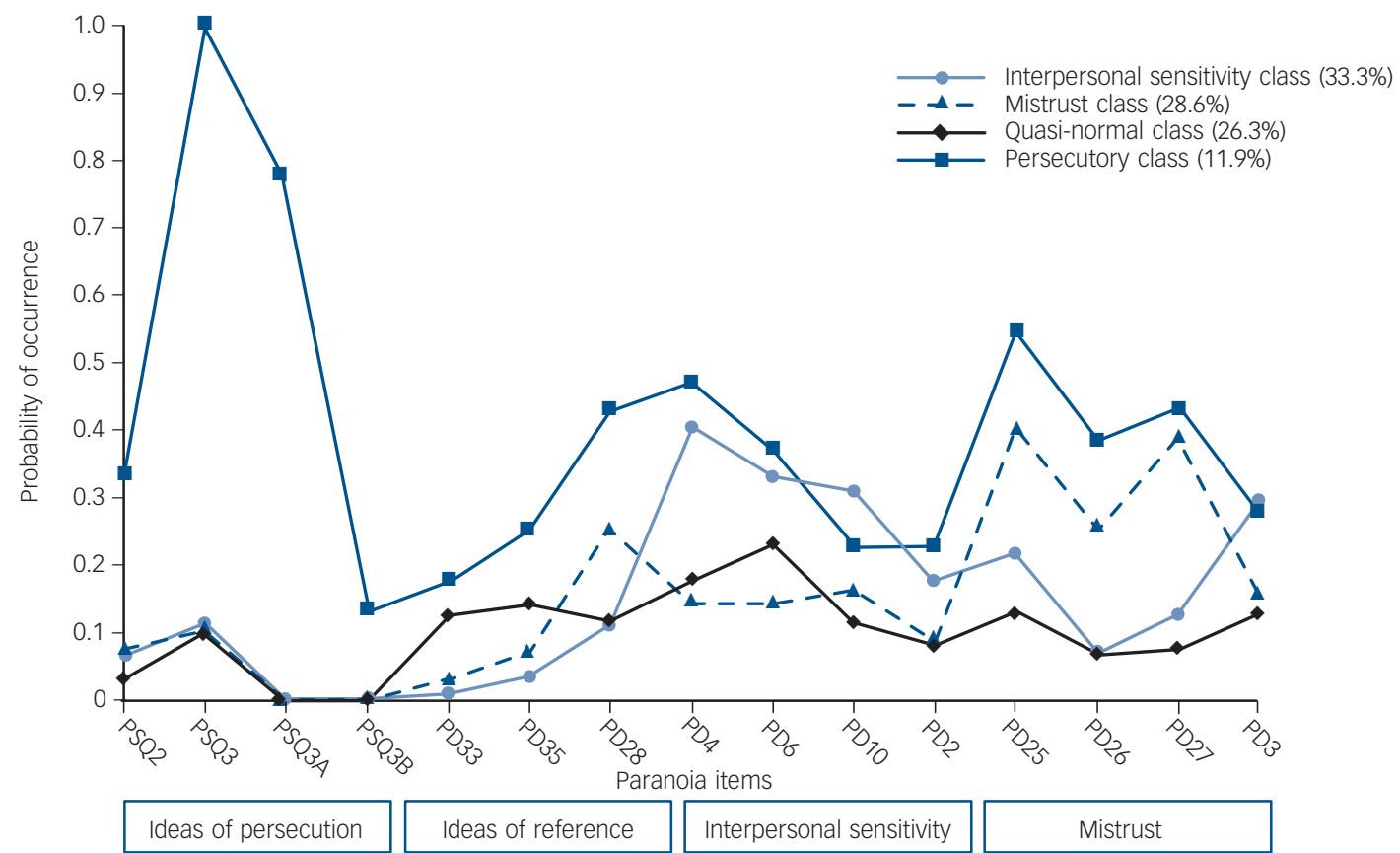

Factors derived from CFA and their item components

Fig. 3 Estimated probabilities for the occurrence of 15 paranoia items in the one-factor four-class mixture model (FMM-4).

CFA, confirmatory factor analysis; PSQ, Psychosis Screening Questionnaire; PD, Structured Clinical Interview for DSM-IV Axis II Disorders, personality disorder. 
Table 4 Results from latent class analysis of 15 paranoia items in the 2000 British National Psychiatric Morbidity Survey ( $n=8576$ )

\begin{tabular}{|c|c|c|c|c|c|c|c|c|}
\hline Model & Log-likelihood & $\begin{array}{c}\text { Replicated } \\
\text { log-likelihood }\end{array}$ & $\begin{array}{c}\text { Free } \\
\text { parameters, } n\end{array}$ & AIC & $\mathrm{BIC}$ & SSABIC & LMR-LRT $(P)$ & Entropy \\
\hline $1 \mathrm{C}$ & -53653.224 & Yes & 15 & 107336.449 & 107442.300 & 107394.632 & NA & NA \\
\hline $2 \mathrm{C}$ & -46637.077 & Yes & 31 & 93336.154 & 93554.912 & 93456.400 & $13936.122(<0.001)$ & 0.849 \\
\hline $3 c$ & -45698.149 & Yes & 47 & 91490.298 & 91821.964 & 91672.606 & $1864.986(<0.001)$ & 0.751 \\
\hline $4 C$ & -44805.585 & Yes & 63 & 89737.169 & 90181.743 & 89981.540 & $1772.894(<0.001)$ & 0.799 \\
\hline $5 c$ & -44467.169 & Yes & 79 & 89092.338 & 89649.819 & 89398.771 & $672.192(<0.001)$ & 0.790 \\
\hline $6 c$ & -44190.940 & Yes & 95 & 88571.880 & 89242.268 & 88940.375 & $548.672(<0.001)$ & 0.785 \\
\hline $7 c$ & -44068.571 & Yes & 111 & 88359.142 & 89142.438 & 88789.700 & 243.061 (0.0138) & 0.795 \\
\hline $8 c$ & -43970.504 & No & 127 & 88195.008 & 89091.211 & 88687.628 & $194.790(0.0265)$ & 0.793 \\
\hline
\end{tabular}

Table 5 Results from factor mixture models (FMM) of 15 paranoia items in the 2000 British National Psychiatric Morbidity

\begin{tabular}{|c|c|c|c|c|c|c|}
\hline Model & Log-likelihood & $\begin{array}{l}\text { Replicated } \\
\text { log-likelihood }\end{array}$ & $\begin{array}{c}\text { Free } \\
\text { parameters, } n\end{array}$ & AIC & $\mathrm{BIC}$ & SSABIC \\
\hline \multicolumn{7}{|c|}{ FMM-1 } \\
\hline $2 \mathrm{C}$ & -46637.077 & Yes & 31 & 93336.154 & 93554.3912 & 93456.400 \\
\hline $3 c$ & -45746.095 & Yes & 33 & 91558.189 & 91791.061 & 91686.193 \\
\hline $4 \mathrm{C}$ & -45619.330 & Yes & 35 & 91308.659 & 91555.645 & 91444.421 \\
\hline $5 c$ & -45593.659 & Yes & 37 & 91261.317 & 91522.416 & 91404.837 \\
\hline \multicolumn{7}{|c|}{ FMM-2 } \\
\hline $2 c$ & -45596.157 & Yes & 33 & 91258.315 & 91491.187 & 91386.319 \\
\hline $3 c$ & -45562.565 & No & 36 & 91197.131 & 91451.173 & 91336.771 \\
\hline $4 C$ & -45594.967 & No & 39 & 91267.934 & 91543.146 & 91419.211 \\
\hline $5 c$ & -45593.219 & No & 42 & 91270.439 & 91566.821 & 91433.353 \\
\hline \multicolumn{7}{|c|}{ FMM-3 } \\
\hline $2 \mathrm{C}$ & -44434.219 & Yes & 46 & 88960.438 & 89285.047 & 89138.867 \\
\hline $3 c$ & -44054.713 & Yes & 62 & 88233.426 & 88670.943 & 88473.918 \\
\hline $4 \mathrm{C}$ & -43919.327 & Yes & 78 & 87994.653 & 88545.078 & 88297.207 \\
\hline $5 c$ & -43846.883 & Yes & 94 & 87881.766 & 88545.098 & 88246.383 \\
\hline \multicolumn{7}{|c|}{ FMM-4 } \\
\hline $2 \mathrm{C}$ & -44433.857 & Yes & 47 & 88961.715 & 89293.381 & 89144.023 \\
\hline $3 c$ & -44052.647 & Yes & 64 & 88233.294 & 88684.924 & 88481.544 \\
\hline $4 c$ & -43917.581 & Yes & 81 & 87997.163 & 88568.758 & 88311.354 \\
\hline $5 c$ & -43844.729 & Yes & 98 & 87885.458 & 88577.016 & 88265.590 \\
\hline \multicolumn{7}{|c|}{ FMM-5 } \\
\hline $2 \mathrm{C}$ & -44419.630 & No & 61 & 88961.261 & 89391.721 & 89197.874 \\
\hline $3 c$ & -43993.243 & No & 92 & 88170.485 & 88819.704 & 88527.344 \\
\hline $4 \mathrm{C}$ & -43846.878 & No & 123 & 87939.756 & 88807.733 & 88416.861 \\
\hline $5 c$ & -43795.600 & No & 154 & 87899.200 & 88985.935 & 88496.551 \\
\hline
\end{tabular}

grouped within the factors tested by the CFA. The three largest classes between them include nearly $90 \%$ of participants (each class accounting for between a quarter and a third), and were characterised by a uniformly low probability of endorsing ideas of persecution. The largest class comprised $33.3 \%$ of the sample, and as a group its members scored highly on interpersonal sensitivity and moderately on mistrust: it is best described as the interpersonal sensitivity class. The second class was almost as common $(28.6 \%)$. It can be termed the mistrust class, as its members scored more highly than the interpersonal sensitivity class on the mistrust items, but lower on interpersonal sensitivity. These two classes both displayed some endorsement of item 28 of the SCID-II in the ideas of reference factor ('Do you often detect hidden threats or insults in things people say or do?'), an item that shares attributes of interpersonal sensitivity. The members of class 3 had roughly equal rates of endorsement of ideas of reference, interpersonal sensitivity, and mistrust. Their endorsement of the items in these three factors was relatively infrequent, varying between $10 \%$ and $20 \%$. Consequently, they scored slightly higher on ideas of reference than the first two classes. Nevertheless, given their relatively low rates of overall endorsement of items, they might reasonably be designated the quasi-normal class. The fourth class was much less common than the first three (11.9\%). It was also much more symptomatic, characterised by a high probability of perceiving direct threats to personal safety (all of them felt people were against them, three-quarters agreed that people were deliberately trying to harm them, and nearly $15 \%$ thought people were plotting against them). It was the only group that scored highly on ideas of persecution, and its endorsement of items from the other three factors was almost invariably higher than that of the other classes. They constitute a clear persecutory class. The 'severity' factor variance for the underlying paranoid ideation dimension was lowest in the mistrust class (0.45), followed by the interpersonal sensitivity class (0.48), then the persecutory class, and finally the quasi-normal class (0.97). 


\section{Discussion}

In this paper we used a secondary analysis of items from the SCID-II questionnaire and the PSQ to identify structural relationships in the spectrum of paranoid ideation. These items have face validity for detecting paranoid ideation, in that, because of the form they take, they seem likely to distinguish both between different thought contents and between people with stronger and weaker paranoid inclinations. They were selected a priori to test our hypotheses and no other items were examined.

Some of the items were endorsed by $20-30 \%$ of the general population. These covered a consciousness of a lack of assertiveness, worries over social inferiority, worries over criticism by others, feelings that people were generally against the respondent and might use or hurt them, and a reluctance to reveal too much in case people used it in adverse ways. Ideas of reference involving the detection of hidden threats or insults were almost as common. A sixth of the population spent a lot of time wondering whether they could trust their friends or work colleagues. About $10 \%$ of the population sometimes felt that people were watching them, staring at them, deliberately acting to harm them or trying to control their thoughts. Slightly fewer felt that people in public places might be talking about them. Finally, a much smaller proportion, but still nearly $2 \%$ of the population, thought that some group was plotting to cause them serious harm or injury.

Our results supported our initial hypothesis that items reflecting paranoid ideation would follow an exponential distribution like that seen with affective symptoms. ${ }^{16}$ Only one other group seems to have approached attributes relating to paranoia in this way, albeit by using conventional categories of personality disorder. ${ }^{31}$ They found a slightly different (cubic) curve of distribution for items forming the diagnostic criteria for paranoid personality disorder (the item count with the highest frequency was 2 rather than 0 ).

Confirmatory factor analysis provided strong evidence in support of our postulated categorisation of paranoid experience, clearly identifying factors representing mistrust, interpersonal sensitivity, ideas of reference, and ideas of persecution. Latent class analysis models offered only an incomplete account of the data. However, LCA does not allow for dimensional aspects of item distribution, and these are very likely to be present. We therefore applied FMMA to our data.

Theoretical considerations are regarded as important in the interpretation of FMMA. The two models of best fit obtained by FMMA both included a provision for variations in overall severity. The model FMM-3/4C, in which variations were permitted within classes, but not between classes, was a slightly better fit, but relied on an implausible assumption incompatible with our initial conceptualisation of paranoia. Thus, on theoretical and empirical grounds, we favoured model FMM-4/4C, which did allow for variation in severity between classes.

In our study, the less frequent items had a content suggestive of a greater disturbance in social perception. Our analyses also support our hypothesis of a non-reflexive relationship: that these less frequent, more severe items were relatively more predictive of other paranoia items. This tallies with the idea that paranoid ideation is continuously distributed, with actual paranoid delusions being placed at the extreme end of the continuum. At a single point in time, the continuum is defined by differences between individuals located at individual positions on the curve. However, people are themselves likely to vary in a way that would place them at different positions on the curve at different times, dependent on changing circumstances. In a sense, they would move along the curve, a speculation now with some support from longitudinal investigation., ${ }^{7,32}$

The continuum model was also supported by the FMMA: although this identifies subcategories of individuals, the rarest class (the only one strongly associated with ideas of persecution) almost invariably had the highest rates of the other features of paranoia. The quasi-normal class had low rates of items forming the mistrust, interpersonal sensitivity, and ideas of reference factors, and showed no endorsement of persecutory ideas. Positioned between these classes were two intermediate classes, loaded particularly towards mistrust and ideas of reference respectively. It is possible that these represent alternative routes into more florid ideas of persecution. In general, our analyses supported the existence both of the subcategories of paranoia and of an underlying dimension. Movement between the categories and along the dimension indicate the processes whereby the more extreme forms of paranoia develop, eventually resulting in diagnosable psychotic disorders.

This investigation, based on a random sample of the general population of Great Britain corroborates our study of paranoia in a student population. ${ }^{3}$ The relative frequency of individual items was similar, and there was a continuous exponential distribution of paranoid thoughts, with similar non-reflexive relationships between less and more frequent items.

\section{Limitations}

There is inevitably a degree of inaccuracy in the methods feasible in large surveys, but this is traded off against the sample size required in a study of the structure of paranoia. However, selfreport items like those used here correlate both with interviewer assessments (e.g. Inacu et $a l^{33}$ ) and with experimental investigations (e.g. Freeman $e a^{34}$ ).

Questions in the PSQ apply to experiences within the past year, while the SCID-II asks about an implicit general tendency to think in particular ways. Thus, the instruments are potentially discrepant in relation to the timing of the experiences they tap. This may not be crucial, as the propensity to paranoid thought may operate as a mixture of trait and state attributes. We were unable to take account of the possibility that the paranoid ideation of individual participants was grounded in reality, although the capacity of the characteristics of experience to elicit paranoia is itself likely to occur on a dimension.

The models were tested on a single sample, and require replication.

\section{Implications}

If we take the rates of endorsement of paranoid items in our study at face value, they suggest that paranoia is so common as to be almost normal. We are certainly obliged to make decisions to trust or to mistrust on a daily basis. The sheer frequency of paranoid beliefs implies that, to some degree, it can be adaptive in social situations. $^{31}$ Individuals who are trusting, open and never suspicious of the intentions of others may end up as naive objects of exploitation. When surrounded by strangers, it may be better to remain somewhat wary of their intentions until they are definitely seen to be favourable. However, too great a degree of suspiciousness may obstruct the development of the social relationships necessary for the maintenance of well-being. ${ }^{35}$ Thus, paranoia leads to isolation that may foster the retention of unusual ideas by removing the possibility of normalising exposure. ${ }^{36}$

Our results have implications for the aetiological study of psychosis. ${ }^{17}$ Other continua are almost certainly involved. ${ }^{5}$ 
Evidence encourages the separate consideration of paranoia, grandiosity, hallucinations and thought disorder (e.g. Wigman et $a b^{37}$ ). Our findings also imply that in some people movement along these continua results in the emergence of psychosis. ${ }^{32}$ Thus, the role of aetiology is to explain exactly why particular people make this journey at particular times in their lives. In the psychological domain, this implies the concatenation of different psychological attributes, some cognitive, some emotional. ${ }^{38,39}$ In social terms, adverse early experiences in increasing the propensity to paranoia, may have a tonic effect on people's position on the curve, whereas more recent events may be responsible for more immediate movement along it. The role of appraisal in this process is likely to be crucial, ${ }^{40-42}$ and offers an opportunity for focused psychological treatments, as does the normalising implications of the widespread distribution of paranoid thought, which may reduce self-stigmatisation. Our findings would also encourage the consideration of treatment at an early stage.

Finally, the continuum model of psychosis has complex implications for diagnostic systems. We would agree with Linscott \& van $\mathrm{Os}^{4}$ that taxonomic classifications of schizophrenia, while remaining of heuristic value, create pragmatic divisions that do not map onto corresponding latent discontinuities. However, they argue that the empirically demonstrated continua of experience probably do have an underlying structure. Our results are a corroboration of their position with the additional advantage of being based on specific theoretical considerations.

Paul E. Bebbington, PhD, FRCP, FRCPsych, Mental Health Sciences Unit, University College London Faculty of Brain Sciences, UK; Orla McBride, PhD, School of Psychology, University of Ulster, Northland Road, Londonderry, Northern Ireland, UK; Craig Steel, PhD, Charlie Waller Institute, School of Psychology and Clinical Language Sciences, University of Reading, UK; Elizabeth Kuipers, BSC, MSC, PhD, Department of Psychology, Institute of Psychiatry, King's College London, and NIHR Biomedical Research Centre, South London and Maudsley NHS Foundation Trust, UK; Mirjana Radovanovič, MD, MSC, University Psychiatric Hospital, Alcoholism Treatment Center, Ljubljana, Slovenia; Traolach Brugha, MD, FRCPsych, Department of Health Sciences, University of Leicester, UK; Rachel Jenkins, MD, FRCPsych, WHO Collaborating Centre, Institute of Psychiatry, Kings College London, UK; Howard Meltzer (deceased), PhD, Department of Health Sciences, University of Leicester, UK Daniel Freeman, PhD, DClinPSy, FBPSS, Department of Psychiatry, Oxford University, Warneford Hospital, Oxford, UK

Correspondence: Professor Paul E. Bebbington, UCL Mental Health Sciences Unit, Charles Bell House, 67-73 Riding House Street, London W1W 7EJ, UK. Email: p.bebbington@ucl.ac.uk

First received 9 Aug 2012, final revision 18 Jan 2013, accepted 4 Mar 2013

\section{Acknowledgement}

Our co-author, colleague and friend Professor Howard Meltzer died on 17 January 2013.

\section{References}

1 van Os J, Verdoux H. Diagnosis and classification of schizophrenia: categories versus dimensions, distributions versus disease. In The Epidemiology of Schizophrenia (eds RM Murray, PB Jones, E Susser, J van Os, M Cannon): 364-410. Cambridge University Press, 2003.

2 Johns LC, Cannon M, Singleton N, Murray RM, Farrell M, Brugha T, et al. Prevalence and correlates of self-reported psychotic symptoms in the British population. Br J Psychiatry 2004; 185: 298-305.

3 Freeman D, Garety PA, Bebbington PE, Smith B, Rollinson R, Fowler D, et al. Psychological investigation of the structure of paranoia in a non-clinical population. Br J Psychiatry 2005; 186: 427-35.

4 Linscott RJ, van Os J. Systematic reviews of categorical versus continuum models in psychosis: evidence for discontinuous subpopulations underlying a psychometric continuum. Implications for DSM-V, DSM-VI, and DSM-VII. Annu Rev Clin Psychol 2010; 6: 391-419.

5 Kaymaz N, van Os J. Extended psychosis phenotype - yes: single continuum - unlikely. Psychol Med 2010; 40: 1963-6.
6 Poulton R, Caspi A, Moffitt TE, Cannon M, Murray R, Harrington H-L. Children's self-reported psychotic symptoms and adult schizophreniform disorder. A 15-year longitudinal study. Arch Gen Psychiatry 2000; 57: 1053-8.

7 Kaymaz N, Drukker M, Lieb R, Wittchen H-U, Werbeloff N, Weiser M, et al. Do subthreshold psychotic experiences predictclinical outcomes in unselected non-help-seeking population-based samples? A systematic review and meta-analysis, enriched with new results. Psychol Med 2012; Jan 20: 1-15. Epub ahead of print.

8 Freeman D, Garety PA, Kuipers E, Fowler D, Bebbington PE. A cognitive model of persecutory delusions. Br J Clin Psychol 2002; 41: 331-47.

9 Freeman D. Suspicious minds: the psychology of persecutory delusions. Clin Psychol Rev 2007; 27: 425-57.

10 Freeman $D$, McManus $S$, Brugha $T$, Meltzer $H$, Jenkins R, Bebbington $P$. Concomitants of paranoia in the general population. Psychol Med 2011; 41: 923-36.

11 Freeman D, Stahl D, McManus S, Meltzer H, Brugha T, Wiles N, et al. Insomnia, worry, anxiety and depression as predictors of the occurrence and persistence of paranoid thinking. Soc Psychiatry Psychiatr Epidemiol 2012; 47: 1195-203.

12 Schutters SI, Dominguez MD, Knappe S, Lieb R, van Os J, Schruers KR, et al. The association between social phobia, social anxiety cognitions and paranoid symptoms. Acta Psychiatr Scand 2012; 125: 213-27.

13 Thewissen V, Bentall RP, Oorschot M, A Campo J, van Lierop T, van Os J, et al. Emotions, self-esteem, and paranoid episodes: an experience sampling study. Br J Clin Psychol 2011; 50: 178-95.

14 Fowler D, Hodgekins J, Garety P, Freeman D, Kuipers E, Dunn G, et al. Negative cognition, depressed mood, and paranoia: a longitudinal pathway analysis using structural equation modelling. Schizophr Bull 2012; 38: 1063-73.

15 Wigman JT, van Nierop M, Vollebergh WA, Lieb R, Beesdo-Baum K, Wittchen $\mathrm{HU}$, et al. Evidence that psychotic symptoms are prevalent in disorders of anxiety and depression, impacting on illness onset, risk, and severity - implications for diagnosis and ultra-high risk research. Schizoph Bull 2012; 38: 247-57.

16 Melzer D, Tom BDM, Brugha TS, Fryers T, Meltzer H. Common mental disorder symptom counts in populations: are there distinct case groups above epidemiological cut-offs? Psychol Med 2002; 32: 1195-201.

17 Plomin R, Haworth CMA, Davis OSP. Common disorders are quantitative traits. Nat Rev Genet 2009; 10: 872-8.

18 Sturt E. Hierarchical patterns in the distribution of psychiatric symptoms. Psychol Med 1981; 11: 783-94.

19 Singleton N, Bumpstead R, O'Brien M, Lee A, Meltzer H. Psychiatric Morbidity Among Adults Living in Private Households. TSO (The Stationery Office), 2001

20 Singleton N, Lee A, Meltzer H. Psychiatric Morbidity Among Adults Living in Private Households: Technical Report. TSO (The Stationery Office), 2001.

21 Kish L. Survey Sampling. Wiley \& Sons, 1965.

22 Bebbington PE, Nayani T. The Psychosis Screening Questionnaire. Int J Methods Psychiatr Res 1995; 5: 11-20.

23 First MB, Gibbon M, Spitzer RL, Williams JBW, Benjamin L. Structured Clinical Interview for DSM-IV Axis II Personality Disorders. American Psychiatric Press, 1997.

24 Jenkins $\mathrm{R}$, Meltzer $\mathrm{H}$, Bebbington $\mathrm{P}$, Brugha $\mathrm{T}$, Farrell M, McManus $\mathrm{S}$, et al. The British Mental Health Survey Programme: achievements and latest findings. Soc Psychiatry Psychiatr Epidemiol 2009; 44: 899-904.

25 Muthén LK, Muthén BO. Mplus User's Guide, 6th edn. Muthén and Muthén, 2010.

26 Hagenaars JA, McCutcheon AL. Advances in Latent Class Models. Cambridge University Press, 2002.

27 Shevlin M, Adamson G, Vollebergh W, de Graaf R, van Os J. An application of item response mixture modelling to psychosis indicators in two large community samples. Soc Psychiatry Psychiatr Epidemiol 2007; 42: 771-9.

28 Lubke GH, Muthén B. Investigating population heterogeneity with factor mixture models. Psychol Methods 2005; 10: 21-39.

29 Clark SL, Muthén B, Kaprio J, D'Onofrio BM, Viken R, Rose RJ, et al. Models and strategies for factor mixture analysis: two examples concerning the structure underlying psychological disorders. Accessed 6 Mar 2013, http:// www.statmodel.com/download/FMA\%20Paper_v142.pdf.

30 Muthén $B$, Asparouhove T. Item response mixture modeling: Application to tobacco dependence criteria. Addict Behav 2006; 31: 1050-66.

31 Lyoo IK, Youn T, Ha TH, Park HS, Kwon JS. Classification of frequency distributions of diagnostic criteria scores in twelve personality disorders by the curve fitting method. Psychiatr Clin Neurosci 2003; 57: 417-23. 
32 Werbeloff N, Drukker M, Dohrenwend BP, Levav I, Yoffe R, van Os J, et al. Self-reported attenuated psychotic symptoms as forerunners of severe mental disorders later in life. Arch Gen Psychiatry 2012; 69: 467-75.

33 Iancu I, Poreh A, Lehman B, Shamir E, Kotler M. The Positive and Negative Symptoms Questionnaire. Compr Psychiatry 2005; 46: 61-6.

34 Freeman $\mathrm{D}$, Pugh $\mathrm{K}$, Antley $\mathrm{A}$, Slater $\mathrm{M}$, Bebbington $\mathrm{P}$, Gittins $\mathrm{M}$, et al. Virtual reality study of paranoid thinking in the general population. Br J Psychiatry 2008; 192: 258-63.

35 Penn DL, Mueser KT, Tarrier N, Gloege A, Cather C, Serrano D, et al Suppportive therapy for schizophrenia: positive mechanism and implications for adjunctive psychosocial treatments. Schizophr Bull 2004; 30: 101-12.

36 White R, Bebbington P, Pearson J, Johnson S, Ellis D. The social context of insight in schizophrenia. Soc Psychiatry Psychiatr Epidemiol 2000; 35 500-7.

37 Wigman JTW, Vollebergh WAM, Raaijmakers QAW, ledema J, van Dorsselaer S, Ormel J, et al. The structure of the extended psychosis phenotype in early adolescence - a cross-sample replication. Schizophr Bull 2011; 37: 850-60.
38 Krabbendam L, Myin-Germeys I, Hanssen M, de Graaf R, Vollebergh W, Bak M, et al. Development of depressed mood predicts onset of psychotic disorder in individuals who report hallucinatory experiences. Br J Clin Psychol 2005; 44: $113-25$

39 Hanssen M, Bak M, Bijl R, Vollebergh W, van Os J. The incidence and outcome of subclinical psychotic experiences in the general population. Br J Clin Psychol 2005; 44: 181-91.

40 Garety P, Kuipers E, Fowler D, Freeman D, Bebbington P. Theoretical paper: a cognitive model of the positive symptoms of psychosis. Psychol Med 2001; 31: 189-95.

41 Garety PA, Bebbington P, Fowler D, Freeman D, Kuipers E. Implications for neurobiological research of cognitive models of psychosis: a theoretical paper. Psychol Med 2007; 37: 1377-91.

42 Morrison AP, French P, Wells A. Metacognitive beliefs across the continuum of psychosis: Comparisons between patients with psychotic disorders, patients at ultra-high risk, and non-patients. Behav Res Ther 2007; 45: 2241-6

\section{The Edge}

poems

by doctors

\section{Charles Montgomery}

They emerge outside the hospital

Dazed by our day,

In dressing-gown and slippers -

The pale ones

Searching the sunlight

For something familiar:

The bright cars and brisk

comings and goings

Of life.

Behind them sliding doors and

Shadows shelve steeply

To the sound of phones;

Shoes squeal on tiles

As routine

Criss-crosses with something more fragile.

And no one knows

Where the edge begins,

This strange overlap,

But all through the day

They gather

At the buildings entrance:

Anonymous filed numbers,

To lean against warm walls

And breathe town air

And to stand blinking away the minutes

Of wide blue spaces,

While the summers dust passes

Unnoticed

In and out of light.

Charles Montgomery, Consultant Psychiatrist, Exeter.

Another of Charles Montgomery's poems, 'Back Ward at Glenside', was published in the Journal in March 2013. 